\title{
A Series Approach to Perturbed Stochastic Volterra Equations of Convolution Type
}

\author{
Anna Karczewska, Bartosz Bandrowski \\ Faculty of Mathematics, Computer Science and Econometrics, University of Zielona Góra, Zielona Góra, Poland \\ Email: A.Karczewska@wmie.uz.zgora.pl
}

Received 6 July 2015; accepted 4 September 2015; published 7 September 2015

Copyright $(2015$ by authors and Scientific Research Publishing Inc.

This work is licensed under the Creative Commons Attribution International License (CC BY). http://creativecommons.org/licenses/by/4.0/

(c) () Open Access

\begin{abstract}
In the paper, perturbed stochastic Volterra Equations with noise terms driven by series of independent scalar Wiener processes are considered. In the study, the resolvent approach to the equations under consideration is used. Sufficient conditions for the existence of strong solution to the class of perturbed stochastic Volterra Equations of convolution type are given. Regularity of stochastic convolution is supplied, as well.
\end{abstract}

\section{Keywords}

Stochastic Linear Volterra Equation, Perturbed Equation, Strong Solution, Resolvent, Mild Solution, Stochastic Convolution, Series Approach

\section{Introduction}

Let $\left(H,|\cdot|_{H}\right)$ be a seperable Hilbert space and let $\left(\Omega, \mathcal{F},(\mathcal{F})_{t \geq 0}, P\right)$ denote a probability space. We consider perturbed stochastic Volterra Equations in $H$ of the form

$$
\begin{aligned}
X(t)= & X_{0}+\int_{0}^{t}[a(t-\tau)+(a \times k)(t-\tau)] A X(\tau) \mathrm{d} \tau \\
& +\int_{0}^{t} b(t-\tau) X(\tau) \mathrm{d} \tau+\sum_{i=1}^{\infty} \int_{0}^{t} \Psi_{i}(\tau) \mathrm{d} W_{i}(\tau), \quad t \geq 0,
\end{aligned}
$$

where $X_{0}$ is an $H$-valued $\mathcal{F}_{0}$-measurable random variable, kernels $a, k, b$ are real valued and locally integrable functions defined on $\mathbb{R}_{+}$and $A$ is a closed unbounded linear operator in $H$ with a dense domain $D(A)$. The domain $D(A)$ is equipped with the graph norm $|\cdot|_{D(A)}$ of $A$, i.e. $|h|_{D(A)}:=\left(|h|_{H}^{2}+|A h|_{H}^{2}\right)^{1 / 2}$. 
In our work, the Equation (1) is driven by series of scalar Wiener processes; $W=\left(W_{i}\right)_{i=1}^{\infty}$ and $\Psi=\left(\Psi_{i}\right)_{i=1}^{\infty}$ are appropriate processes defined below.

The goal of this paper is to formulate sufficient conditions for the existence and regularity of strong solutions to the perturbed Volterra Equation driven by series of scalar Wiener processes. Previously, in [1]-[4], the stochastic integral for Hilbert-Schmidt operator-valued integrands and Wiener processes with values in Hilbert space has been constructed. Moreover, the particular series expansion of the Wiener process with respect to the eigenvectors of its covariance operator has been used. The stochastic integral used in this paper, originally introduced in [5], bases on the construction directly in terms of the sequence of independent scalar processes. In consequence, the stochastic integral is independent of any covariance operator usually connected with a noise process.

In the paper, we use the resolvent approach to the Equation (1). This means that a deterministic counterpart of the Equation (1), that is, the Equation

$$
u(t)=f(t)+\int_{0}^{t}[a(t-\tau)+(a \times k)(t-\tau)] A u(\tau) \mathrm{d} \tau+\int_{0}^{t} b(t-\tau) u(\tau) \mathrm{d} \tau,
$$

admits a resolvent family. In (2), the operator $A$ and the kernel functions are the same as previously in (1) and $f$ is a $H$-valued function.

By $R(t), t \geq 0$, we shall denote the family of resolvent operators corresponding to the Volterra Equation (2), which is defined as follows.

Definition 1 A family $\{R(t)\}_{t \geq 0}$ of bounded linear operators in $H$ is called resolvent for (2), if the following conditions are satisfied:

1) $R(t)$ is strongly continuous on $\mathbb{R}_{+}$and $R(0)=I$;

2) $R(t)$ commutes with the operator $A$, that is, $R(t)(D(A)) \subset D(A)$ and $A R(t) x=R(t) A x$ for all $x \in D(A), t \geq 0$;

3) the following resolvent equation holds

$$
R(t) x=x+\int_{0}^{t}[a(t-\tau)+(a \times k)(t-\tau)] A R(\tau) x \mathrm{~d} \tau+\int_{0}^{t} b(t-\tau) R(\tau) x \mathrm{~d} \tau,
$$

for all $x \in D(A), t \geq 0$.

In this paper, the following result concerning convergence of resolvents for the Equation (1) will play the key role.

As in [6], we shall assume the following hypotheses:

$\left(\mathbf{H}_{\alpha}\right)$ The solution of the Equation

$$
\alpha(t)=(\alpha \times b)(t)+(a \times k)(t)+a(t), t \geq 0,
$$

is nonnegative, nonincreasing and convex.

$\left(\mathbf{H}_{\beta}\right)$ The solution of the Equation $\beta(t)=1+(\beta \times b)(t), t \geq 0$, is differentiable.

Theorem 1 ([6], Th. 3.5) Assume that $A$ is the generator of bounded analytic semigroup of $H$. Suppose that the hypotheses $\left(\mathbf{H}_{\alpha}\right)$ and $\left(\mathbf{H}_{\beta}\right)$ are satisfied. Then the Equation (2) admits a resolvent $\{R(t)\}_{t \geq 0}$. Additionally, there exists bounded operators $A_{n}$ and corresponding resolvent families $\left\{R_{n}(t)\right\}_{t \geq 0}$ satisfying $\left\|R_{n}(t)\right\| \leq M \beta(t)$ for all $t>0, n \in \mathbb{N}$, such that

$$
R_{n}(t) x \rightarrow R(t) x, \text { gdy } n \rightarrow \infty
$$

for all $x \in H, t \geq 0$. Moreover, the convergence (4) is uniform in t on every compact subset of $\mathbb{R}_{+}$.

Below we give an example illustrating conditions $\left(\mathbf{H}_{\alpha}\right)$ and $\left(\mathbf{H}_{\beta}\right)$.

Example 1 Consider in the Equation (1) the following kernel functions

$$
a(t) \equiv 1, \quad b(t) \equiv 0, k(t)= \begin{cases}2 t-2 & \text { for } t \leq 1 \\ 0 & \text { for } t>1\end{cases}
$$

Then the functions

$$
\alpha(t)=\left\{\begin{array}{ll}
(t-1)^{2} & \text { for } t \leq 1 \\
0 & \text { for } t>1
\end{array} \text { and } \beta(t) \equiv 1\right.
$$


fulfil conditions $\left(\mathbf{H}_{\beta}\right)$ and $\left(\mathbf{H}_{\beta}\right)$.

The paper is organized as follows. Section 2 constains the construction of the stochastic integral due to $\mathrm{O}$. van Gaans [5]. In Section 3, we compare mild and weak solutions and then we provide sufficient conditions for stochastic convolution to be a strong solution to the Equation (1). Section 4 gives regularity of stochastic convolution arising in perturbed Volterra Equation while in Section 5 we derive the analogue of Itô formula to the perturbed Volterra Equation.

\section{The Stochastic Integral}

In this section we recall the construction of the stochastic integral due to O. van Gaans [5].

Definition 2 A function $f:[0, T] \rightarrow L^{2}(\Omega ; H)$ is called piecewise uniformly continuous (PUC) if there are $0=a_{0}<a_{1}<\ldots<a_{n}=T$ such that $f$ is uniformly continuous on $\left(a_{k-1}, a_{k}\right)$ for each $k \in\{1,2, \cdots, n\}$.

Definition 3 A function $f:[0, \infty) \rightarrow L^{2}(\Omega ; H)$ is called piecewise uniformly continuous (PUC), if $\left.f\right|_{[0, T]}$ is uniformly continuous for all $T>0$.

Theorem 2 ([5]) Assume that $\left(W_{i}\right)_{i=1}^{\infty}$ is a series of independent standard scalar Wiener processes with respect to the filtration $\left(\mathcal{F}_{t}\right)_{t \geq 0}$ in $\mathcal{F}$. Let $\left(\Psi_{i}\right)_{i=1}^{\infty}$ be a series of piecewise uniformly continuous functions (PUC) acting from $[0, T]$ into $L^{2}(\Omega ; H)$, adapted with respect to the filtration $\left(\mathcal{F}_{t}\right)_{t \geq 0}$. Then the following results hold.

1) For any $i \in \mathbb{N}$, the integral $\int_{0}^{T} \Psi_{i}(\tau) \mathrm{d} W_{i}(\tau)$ is well-defined as the limit of Riemann sums $L^{2}(\Omega ; H)$ of the form

$$
\sum_{k=1}^{n} \Psi_{i}\left(\tau_{k-1}\right)\left(W_{i}\left(\tau_{k}\right)-W_{i}\left(\tau_{k-1}\right)\right)
$$

where $0=\tau_{0}<\tau_{1}<\ldots<\tau_{n}=T$.

2) For each $i \in \mathbb{N}$, the Itô isometry holds

$$
\mathbb{E}\left|\int_{0}^{T} \Psi_{i}(\tau) \mathrm{d} W_{i}(\tau)\right|_{H}^{2}=\int_{0}^{T} \mathbb{E}\left|\Psi_{i}(\tau)\right|_{L^{2}(\Omega ; H)}^{2} \mathrm{~d} \tau .
$$

3) For any $i, j \in \mathbb{N}$, such that $i \neq j$ we have

$$
\mathbb{E}\left\langle\int_{0}^{T} \Psi_{i}(\tau) \mathrm{d} W_{i}(\tau), \int_{0}^{T} \Psi_{j}(\tau) \mathrm{d} W_{j}(\tau)\right\rangle_{H}=0 .
$$

Definition 4 By $\mathcal{P U C}\left([0, T] ; L^{2}(\Omega ; H)\right)$ we shall denote the space of series $\Psi=\left(\Psi_{i}\right)_{i=1}^{\infty}$ of piecewise uniformly continuous functions (PUC) acting from $[0, T]$ into $L^{2}(\Omega ; H)$, adapted with respect to the filtration $\left(\mathcal{F}_{t}\right)_{t \geq 0}$, such that

$$
\int_{0}^{T} \sum_{i=1}^{\infty} \mathbb{E}\left|\Psi_{i}(\tau)\right|_{L^{2}(\Omega ; H)}^{2} \mathrm{~d} \tau<\infty .
$$

Theorem 3 ([5]) Assume that $W=\left(W_{i}\right)_{i=1}^{\infty}$ is a series of independent standard scalar Wiener processes with respect to the filtration $\left(\mathcal{F}_{t}\right)_{t \geq 0}$ in $\mathcal{F}$. Let $\Psi \in \mathcal{P U C}\left([0, T] ; L^{2}(\Omega ; H)\right)$. Then the integral

exists in $L^{2}(\Omega ; H)$ and

$$
\int_{0}^{T} \Psi(\tau) \mathrm{d} W(\tau):=\sum_{i=1}^{\infty} \int_{0}^{T} \Psi_{i}(\tau) \mathrm{d} W_{i}(\tau)
$$

$$
\mathbb{E}\left|\int_{0}^{T} \Psi(\tau) \mathrm{d} W(\tau)\right|_{H}^{2}=\int_{0}^{T} \mathbb{E} \sum_{i=1}^{\infty}\left|\Psi_{i}(\tau)\right|_{L^{2}(\Omega ; H)}^{2} \mathrm{~d} \tau
$$

\section{The Main Results}

We begin this section with definitions of solutions to the Equation (1). 
Definition 5 An $H$-valued predictable process $X(t), t \in[0, T]$, is said to be a strong solution to (1), if $X$ has a version such that $P(X(t) \in D(A))=1$ for almost all $t \in[0, T]$; for any $t \in[0, T]$

$$
\begin{aligned}
& \int_{0}^{t}|[a(t-\tau)+(a \times k)(t-\tau)] A X(\tau)|_{H} \mathrm{~d} \tau<\infty, P-a . s . \\
& \int_{0}^{t}|b(t-\tau) X(\tau)|_{H} \mathrm{~d} \tau<\infty, P-a . s .
\end{aligned}
$$

and for any $t \in[0, T]$ the Equation (1) holds $P$ - a.s.

Let $A^{*}$ denote the adjoint of $A$ with a dense domain $D\left(A^{*}\right) \subset H$ and the graph norm ||$_{D\left(A^{*}\right)}$.

Definition 6 An $H$-valued predictable process $X(t), t \in[0, T]$, is said to be a weak solution to (1), if

$$
\begin{gathered}
P\left(\int_{0}^{t}|[a(t-\tau)+(a \times k)(t-\tau)] X(\tau)|_{H} \mathrm{~d} \tau<\infty\right)=1, \\
P\left(\int_{0}^{t}|b(t-\tau) X(\tau)|_{H} \mathrm{~d} \tau<\infty\right)=1
\end{gathered}
$$

and if for all $\xi \in D\left(A^{*}\right)$ and all $t \in[0, T]$ the following equation holds

$$
\begin{aligned}
\langle X(t), \xi\rangle_{H}= & \left\langle X_{0}, \xi\right\rangle_{H}+\left\langle\int_{0}^{t}[a(t-\tau)+(a \times k)(t-\tau)] X(\tau) \mathrm{d} \tau, A^{*} \xi\right\rangle_{H} \\
& +\left\langle\int_{0}^{t} b(t-\tau) X(\tau) \mathrm{d} \tau, \xi\right\rangle_{H}+\left\langle\sum_{i=1}^{\infty} \int_{0}^{t} \Psi_{i}(\tau) \mathrm{d} W_{i}(\tau), \xi\right\rangle_{H}, P-\text { a.s. }
\end{aligned}
$$

As we have already written, in the paper we assume that (2) admits a resolvent family $R(t), t \geq 0$. So, we can introduce the following idea.

Definition 7 An $H$-valued predictable process $X(t), t \in[0, T]$, is said to be a mild solution to the perturbed stochastic Volterra Equation (1), if

and, for all $t \in[0, T]$,

$$
\int_{0}^{t} \sum_{i=1}^{\infty} \mathbb{E}\left|R(t-\tau) \Psi_{i}(\tau)\right|_{L^{2}(\Omega ; H)}^{2} \mathrm{~d} \tau<\infty \text { dla } t \leq T
$$

$$
X(t)=R(t) X_{0}+\sum_{i=1}^{\infty} \int_{0}^{t} R(t-\tau) \Psi_{i}(\tau) \mathrm{d} W_{i}(\tau), P-\text { a.s. }
$$

where $\{R(t)\}_{t \geq 0}$ is the resolvent for the deterministic perturbed Volterra Equation (2).

We introduce the stochastic convolution

$$
W^{\Psi}(t):=\sum_{i=1}^{\infty} \int_{0}^{t} R(t-\tau) \Psi_{i}(\tau) \mathrm{d} W_{i}(\tau), t \in[0, T]
$$

where $\Psi$ and the resolvent operators $R(t), t \geq 0$, are the same as above.

Let us formulate some auxiliary results concerning the convolution $W^{\Psi}$.

Proposition 4 For arbitrary process $\Psi \in \mathcal{P U C}\left([0, T] ; L^{2}(\Omega ; H)\right)$, the process $W^{\Psi}(t), t \geq 0$, given by (8) has a predictable version.

Proposition 5 Assume that $\Psi \in \mathcal{P U C}\left([0, T] ; L^{2}(\Omega ; H)\right)$. Then the process $W^{\Psi}(t), t \geq 0$, defined by (8) has square integrable trajectories.

For the idea of proofs of Propositions 4 and 5, we refer to [2] or [3].

In some cases, weak solutions of Equation (1) coincides with mild solutions of (1), see e.g. [2] or [3]. In consequence, having results for the convolution (8) we obtain results for weak solutions.

Proposition 6 Assume that $\Psi \in \mathcal{P U C}\left([0, T] ; L^{2}(\Omega ; H)\right)$. Then the stochastic convolution $W^{\Psi}(t), t \geq 0$, is a weak solution to the Equation (1). 
Hence, we are able to conclude the following result.

Corollary 1 Let A be a linear bounded operator in $H$. If $\Psi \in \mathcal{P U C}\left([0, T] ; L^{2}(\Omega ; H)\right)$, then

$$
\begin{aligned}
W^{\Psi}(t)= & \int_{0}^{t}[a(t-\tau)+(a \times k)(t-\tau)] A W^{\Psi}(\tau) \mathrm{d} \tau \\
& +\int_{0}^{t} b(t-\tau) W^{\Psi}(\tau) \mathrm{d} \tau+\sum_{i=1}^{\infty} \int_{0}^{t} \Psi_{i}(\tau) \mathrm{d} W_{i}(\tau), P-a . s .
\end{aligned}
$$

The formula (9) says that the convolution $W^{\Psi}$ is a strong solution to (1) if the operator A is bounded.

Here we provide sufficient conditions under which the stochastic convolution $W^{\Psi}(t), t \geq 0$, defined by (8) is a strong solution to the Equation (1).

Lemma 1 Assume that $\Phi \in \mathcal{P U C}\left([0, T] ; L^{2}(\Omega ; D(A))\right)$ and $A \Phi \in \mathcal{P U C}\left([0, T] ; L^{2}(\Omega ; H)\right)$. Then

$$
P\left(\sum_{i=1}^{\infty} \int_{0}^{T} \Phi_{i}(t) \mathrm{d} W_{i}(t) \in D(A)\right)=1
$$

and

$$
A \sum_{i=1}^{\infty} \int_{0}^{T} \Phi_{i}(t) \mathrm{d} W_{i}(t)=\sum_{i=1}^{\infty} \int_{0}^{T} A \Phi_{i}(t) \mathrm{d} W_{i}(t), P-\text { a.s. }
$$

Proof Because $\left(D(A),\left.\cdot\right|_{D(A)}\right)$ is a Hilbert space, then the integral

$$
\int_{0}^{T} \Phi(t) \mathrm{d} W(t):=\sum_{i=1}^{\infty} \int_{0}^{T} \Phi_{i}(t) \mathrm{d} W_{i}(t)
$$

exists in $L^{2}(\Omega ; D(A))$ by Theorem 3 .

Denote by $\left(\tilde{s}_{n}\right)_{n=1}^{\infty}$ division of the interval $[0, T]$, that $0=s_{0}<s_{1}<\cdots<s_{n}=T$, $\lim _{n \rightarrow \infty i \in\{1, \ldots, n\}}\left|s_{i}-s_{i-1}\right|=0$. From the definition of the integral and closedness of the operator $A$ we have

$$
\begin{aligned}
\sum_{i=1}^{\infty} \int_{0}^{T} A \Phi_{i}(t) \mathrm{d} W_{i}(t) & =\sum_{i=1}^{\infty} \lim _{m \rightarrow \infty} A \Phi_{i}\left(s_{n}\right)\left(W_{i}\left(s_{n}\right)-W_{i}\left(s_{n-1}\right)\right)=\lim _{m \rightarrow \infty} \sum_{i=1}^{m} \lim _{m \rightarrow \infty} A \Phi_{i}\left(s_{n}\right)\left(W_{i}\left(s_{n}\right)-W_{i}\left(s_{n-1}\right)\right) \\
= & A \lim _{m \rightarrow \infty} \sum_{i=1}^{m} \lim _{n \rightarrow \infty} \Phi_{i}\left(s_{n}\right)\left(W_{i}\left(s_{n}\right)-W_{i}\left(s_{n-1}\right)\right)=A \lim _{m \rightarrow \infty} \sum_{i=1}^{m} \int_{0}^{T} \Phi_{i}(t) \mathrm{d} W_{i}(t) \\
= & A \sum_{i=1}^{\infty} \int_{0}^{T} \Phi_{i}(t) \mathrm{d} W_{i}(t), \quad P-\text { a.s. }
\end{aligned}
$$

Theorem 7 Let A be a closed linear unbounded operator with the dense domain $D(A)$ equipped with the graph norm $\left.\right|_{D(A)}$. Suppose that assumptions of Theorem 1 hold. If $\Psi \in \mathcal{P U C}\left([0, T] ; L^{2}(\Omega ; D(A))\right)$ and $A \Psi \in \mathcal{P U C}\left([0, T] ; L^{2}(\Omega ; H)\right)$, then the stochastic convolution $W^{\Psi}$ is a strong solution to the perturbed stochastic Volterra Equation (1).

Proof Since closed unbounded linear operator $A$ becomes bounded in $\left(D(A),|\cdot|_{D(A)}\right)$, we have

$A W^{\Psi}(\cdot) \in L^{1}([0, T] ; H), P-a$.s. Then from the properties of stochastic convolution we obtain integrability of $[a(T-\cdot)+(a \times k)(T-\cdot)] A W^{\Psi}(\cdot)$ and $b(T-\cdot) W^{\Psi}(\cdot)$. Therefore, conditions (5) and (6) from the definition of strong solution to Equation (1) hold.

It remains to show that the Equation (1) holds $P$ - a.s., i.e.

$$
\begin{aligned}
W^{\Psi}(t)= & \int_{0}^{t}[a(t-\tau)+(a \times k)(t-\tau)] A W^{\Psi}(\tau) \mathrm{d} \tau \\
& +\int_{0}^{t} b(t-\tau) W^{\Psi}(\tau) \mathrm{d} \tau+\sum_{i=1}^{\infty} \int_{0}^{t} \Psi_{i}(\tau) \mathrm{d} W_{i}(\tau), P-a . s .
\end{aligned}
$$


Because the formula (9) holds for any bounded operator, then it holds for the Yosida approximation $A_{n}$ of the operator $A$, too. Then we have

$$
\begin{aligned}
W_{n}^{\Psi}(t)= & \int_{0}^{t}[a(t-\tau)+(a \times k)(t-\tau)] A_{n} W_{n}^{\Psi}(\tau) \mathrm{d} \tau \\
& +\int_{0}^{t} b(t-\tau) W_{n}^{\Psi}(\tau) \mathrm{d} \tau+\sum_{i=1}^{\infty} \int_{0}^{t} \Psi_{i}(\tau) \mathrm{d} W_{i}(\tau), \quad P-a . s .
\end{aligned}
$$

where

$$
W_{n}^{\Psi}(t):=\sum_{i=1}^{\infty} \int_{0}^{t} R_{n}(t-\tau) \Psi_{i}(\tau) \mathrm{d} W_{i}(\tau)
$$

and

$$
A_{n} W_{n}^{\Psi}(t):=A_{n} \sum_{i=1}^{\infty} \int_{0}^{t} R_{n}(t-\tau) \Psi_{i}(\tau) \mathrm{d} W_{i}(\tau) .
$$

To prove that (11) holds, we need to show the following convergences

$$
\lim _{n \rightarrow \infty} \sup _{t \in[0, T]} \mathbb{E}\left|W_{n}^{\Psi}(t)-W^{\Psi}(t)\right|_{H}^{2}=0
$$

and

$$
\lim _{n \rightarrow \infty} \sup _{t \in[0, T]} \mathbb{E}\left|A_{n} W_{n}^{\Psi}(t)-A W^{\Psi}(t)\right|_{H}^{2}=0 .
$$

By assumption $\Psi \in \mathcal{P U C}\left([0, T] ; L^{2}(\Omega ; D(A))\right) \subset \mathcal{P U C}\left([0, T] ; L^{2}(\Omega ; H)\right)$. Because the operators $R_{n}(t)$ are deterministic and bounded for any $t \in[0, T], n \in \mathbb{N}$, then $R_{n}(t-.) \Psi(\cdot)$ belong to $\mathcal{P U C}\left([0, T] ; L^{2}(\Omega ; H)\right)$, too. Hence, the difference

$$
\Delta_{n}(t-\cdot):=R_{n}(t-\cdot) \Psi(\cdot)-R(t-\cdot) \Psi(\cdot)
$$

belongs to $\mathcal{P U C}\left([0, T] ; L^{2}(\Omega ; H)\right)$ for every $t \in[0, T]$ and $n \in \mathbb{N}$. This means that

$$
\int_{0}^{t} \sum_{i=1}^{\infty} \mathbb{E}\left|\Delta_{n, i}(t-\tau)\right|_{L^{2}(\Omega ; H)}^{2} \mathrm{~d} \tau<\infty \text { dla } t \in[0, T]
$$

From the definition of stochastic integral (Theorem 3), for $t \in[0, T]$, we have

$$
\mathbb{E}\left|\int_{0}^{t} \Delta_{n}(t-\tau) \mathrm{d} W(\tau)\right|_{H}^{2}=\int_{0}^{t} \mathbb{E} \sum_{i=1}^{\infty}\left|\Delta_{n, i}(t-\tau)\right|_{L^{2}(\Omega ; H)}^{2} \mathrm{~d} \tau<\infty .
$$

By Theorem 1, the convergence of the resolvent families is uniform with respect to $t$ on every closed intervals, particularly on $[0, T]$. Then we have

$$
\int_{0}^{T} \mathbb{E} \sum_{i=1}^{\infty}\left|\left[R_{n}(T-\tau)-R(T-\tau)\right] \Psi_{i}(\tau)\right|_{L^{2}(\Omega ; H)}^{2} \mathrm{~d} \tau \rightarrow 0 \text { dla } n \rightarrow \infty .
$$

Summing up the above considerations, we obtain

$$
\begin{aligned}
& \sup _{t \in[0, T]} \mathbb{E}\left|\int_{0}^{t} \Delta_{n}(t-\tau) \mathrm{d} W(\tau)\right|_{H}^{2} \equiv \sup _{t \in[0, T]} \mathbb{E}\left|\int_{0}^{t}\left[R_{n}(t-\tau)-R(t-\tau)\right] \Psi(\tau) \mathrm{d} W(\tau)\right|_{H}^{2} \\
& =\sup _{t \in[0, T]} \mathbb{E}\left|\sum_{i=1}^{\infty} \int_{0}^{t}\left[R_{n}(t-\tau)-R(t-\tau)\right] \Psi_{i}(\tau) \mathrm{d} W_{i}(\tau)\right|_{H}^{2} \leq \mathbb{E}\left|\sum_{i=1}^{\infty} \int_{0}^{T}\left[R_{n}(T-\tau)-R(T-\tau)\right] \Psi_{i}(\tau) \mathrm{d} W_{i}(\tau)\right|_{H} \\
& =\int_{0}^{T} \mathbb{E} \sum_{i=1}^{\infty}\left|\left[R_{n}(T-\tau)-R(T-\tau)\right] \Psi_{i}(\tau)\right|_{L^{2}(\Omega ; H)}^{2} \mathrm{~d} \tau \rightarrow 0
\end{aligned}
$$


as $n \rightarrow \infty$. Then, by the dominated convergence theorem the convergence (12) holds.

From the fact that $\Psi \in \mathcal{P U C}\left([0, T] ; L^{2}(\Omega ; D(A))\right)$ and $R(t)(D(A)) \subset D(A)$ we have $R(t-\cdot) \Psi(\cdot) \in \mathcal{P U C}\left([0, T] ; L^{2}(\Omega ; D(A))\right)$. Then, by Lemma $1, P\left(W^{\Psi}(t) \in D(A)\right)=1$.

For any $n \in \mathbb{N}, t \geq 0$, we can write

$$
\left|A_{n} W_{n}^{\Psi}(t)-A W^{\Psi}(t)\right|_{H} \leq\left|A_{n} W_{n}^{\Psi}(t)-A_{n} W^{\Psi}(t)\right|_{H}+\left|A_{n} W^{\Psi}(t)-A W^{\Psi}(t)\right|_{H} .
$$

Then

$$
\begin{aligned}
& \left|A_{n} W_{n}^{\Psi}(t)-A W^{\Psi}(t)\right|_{H}^{2} \leq\left|A_{n} W_{n}^{\Psi}(t)-A_{n} W^{\Psi}(t)\right|_{H}^{2}+\left|A_{n} W^{\Psi}(t)-A W^{\Psi}(t)\right|_{H}^{2} \\
& +2\left|A_{n} W_{n}^{\Psi}(t)-A_{n} W^{\Psi}(t)\right|_{H}\left|A_{n} W^{\Psi}(t)-A W^{\Psi}(t)\right|_{H} \\
& \leq 3\left[\left|A_{n} W_{n}^{\Psi}(t)-A_{n} W^{\Psi}(t)\right|_{H}^{2}+\left|A_{n} W^{\Psi}(t)-A W^{\Psi}(t)\right|_{H}^{2}\right] .
\end{aligned}
$$

To prove that the convergence (13) holds, we need to show that

$$
\lim _{n \rightarrow \infty} \sup _{t \in[0, T]} \mathbb{E}\left|A_{n} W_{n}^{\Psi}(t)-A_{n} W^{\Psi}(t)\right|_{H}^{2}=0
$$

and

$$
\lim _{n \rightarrow \infty} \sup _{t \in[0, T]} \mathbb{E}\left|A_{n} W^{\Psi}(t)-A W^{\Psi}(t)\right|_{H}^{2}=0 .
$$

We shall study the term $\left|A_{n} W_{n}^{\Psi}(t)-A_{n} W^{\Psi}(t)\right|_{H}^{2}$ first. Because the operator $A$ generates a semigroup, we can use the following property of the Yosida approximation

$$
A_{n} x=J_{n} A x \text { dla dowolnych } x \in D(A), \sup _{n}\left\|J_{n}\right\|<\infty,
$$

where $A_{n} x=n A \mathcal{R}(n, A) x=A J_{n} x$ for any $x \in H, \quad J_{n}:=n \mathcal{R}(n, A)$.

Moreover

$$
\begin{array}{ll}
\lim _{n \rightarrow \infty} J_{n} x=x & \text { for every } x \in H, \\
\lim _{n \rightarrow \infty} A_{n} x=A x & \text { for every } x \in D(A) .
\end{array}
$$

For any big enough $n$ and any $x \in D(A)$, we have $A R_{n}(t) x=R_{n}(t) A x$.

Next, by Lemma 1 and closedness of the operator $A$

$$
\begin{aligned}
A_{n} W_{n}^{\Psi}(t) & \equiv A_{n} \sum_{i=1}^{\infty} \int_{0}^{t} R_{n}(t-\tau) \Psi_{i}(\tau) \mathrm{d} W_{i}(\tau) \\
& =J_{n} \sum_{i=1}^{\infty} \int_{0}^{t} A R_{n}(t-\tau) \Psi_{i}(\tau) \mathrm{d} W_{i}(\tau)=J_{n}\left[\sum_{i=1}^{\infty} \int_{0}^{t} R_{n}(t-\tau) A \Psi_{i}(\tau) \mathrm{d} W_{i}(\tau)\right] .
\end{aligned}
$$

Analogously, we have

$$
A_{n} W^{\Psi}(t)=J_{n}\left[\sum_{i=1}^{\infty} \int_{0}^{t} R(t-\tau) A \Psi_{i}(\tau) \mathrm{d} W_{i}(\tau)\right]
$$

Using (19), we receive

$$
\begin{aligned}
& \left|A_{n} W_{n}^{\Psi}(t)-A_{n} W^{\Psi}(t)\right|_{H}=\left|J_{n} \sum_{i=1}^{\infty} \int_{0}^{t}\left[R_{n}(t-\tau)-R(t-\tau)\right] A \Psi_{i}(\tau) \mathrm{d} W_{i}(\tau)\right|_{H} \\
& \leq \sup _{n}\left\|J_{n}\right\| \cdot\left|\sum_{i=1}^{\infty} \int_{0}^{t}\left[R_{n}(t-\tau)-R(t-\tau)\right] A \Psi_{i}(\tau) \mathrm{d} W_{i}(\tau)\right|_{H} .
\end{aligned}
$$

From assumption $A \Psi \in \mathcal{P U C}\left([0, T] ; L^{2}(\Omega ; H)\right)$, so the term $\left[R_{n}(t-\cdot)-R(t-\cdot)\right] A \Psi(\cdot)$ may be treated like 
the difference $\Delta_{n}$ defined by (14).

Then, using (19) and (12), we obtain (17).

For the term $\left|A_{n} W^{\Psi}(t)-A W^{\Psi}(t)\right|_{H}^{2}$ we can repeat the proof of the convergence (12).

$$
\begin{aligned}
\left|A_{n} W^{\Psi}(t)-A W^{\Psi}(t)\right|_{H} & \equiv\left|A_{n} \sum_{i=1}^{\infty} \int_{0}^{t} R(t-\tau) \Psi_{i}(\tau) \mathrm{d} W_{i}(\tau)-A \sum_{i=1}^{\infty} \int_{0}^{t} R(t-\tau) \Psi_{i}(\tau) \mathrm{d} W_{i}(\tau)\right|_{H} \\
& =\left|\sum_{i=1}^{\infty} \int_{0}^{t}\left[A_{n}-A\right] R(t-\tau) \Psi_{i}(\tau) \mathrm{d} W_{i}(\tau)\right|_{H} .
\end{aligned}
$$

By assumption $\Psi, A \Psi \in \mathcal{P U C}\left([0, T] ; L^{2}(\Omega ; H)\right)$. Because $A_{n}$ and $R(t), t \geq 0$, are bounded, so $A_{n} R(t-\cdot) \Psi(\cdot) \in \mathcal{P U C}\left([0, T] ; L^{2}(\Omega ; H)\right)$.

Analogously, $A R(t-\cdot) \Psi(\cdot)=R(t-\cdot) A \Psi(\cdot) \in \mathcal{P U C}\left([0, T] ; L^{2}(\Omega ; H)\right)$.

Since $\left[A_{n}-A\right] R(t-\cdot) \Psi(\cdot) \in \mathcal{P U C}\left([0, T] ; L^{2}(\Omega ; H)\right)$, this term can be treated like the difference $\Delta_{n}$ defined by (14). Hence, for any $t \in[0, T]$, we may write

$$
\begin{aligned}
& \mathbb{E}\left|A_{n} W^{\Psi}(t)-A W^{\Psi}(t)\right|_{H}^{2}=\mathbb{E}\left|\sum_{i=1}^{\infty} \int_{0}^{t}\left[A_{n}-A\right] R(t-\tau) \Psi_{i}(\tau) \mathrm{d} W_{i}(\tau)\right|_{H}^{2} \\
& \leq \int_{0}^{T} \mathbb{E} \sum_{i=1}^{\infty}\left|\left[A_{n}-A\right] R(T-\tau) \Psi_{i}(\tau)\right|_{L^{2}(\Omega ; H)}^{2} \mathrm{~d} \tau<\infty .
\end{aligned}
$$

Using the convergence (20), we have

$$
\int_{0}^{T} \mathbb{E} \sum_{i=1}^{\infty}\left|\left[A_{n}-A\right] R(T-\tau) \Psi_{i}(\tau)\right|_{L^{2}(\Omega ; H)}^{2} \mathrm{~d} \tau \rightarrow 0 \text { dla } n \rightarrow \infty .
$$

Therefore the convergence (18) holds.

\section{Continuity of Trajectories}

In this section, we give sufficient conditions for the continuity of trajectories of the stochastic convolution when the kernel function $b \equiv 0$. Thus, we study the stochastic convolution corresponding to the equation

$$
X(t)=X_{0}+\int_{0}^{t}[a(t-\tau)+(a \times k)(t-\tau)] A X(\tau) \mathrm{d} \tau+\sum_{i=1}^{\infty} \int_{0}^{t} \Psi_{i}(\tau) \mathrm{d} W_{i}(\tau),
$$

where $t \in[0, T]$.

Theorem 8 Let the operator A be the generator of strongly continuous bounded analytic semigroup $T(t)$, $t \in[0, T]$. Assume that the functions $a, \dot{a}, k \in L_{\text {loc }}^{1}([0, T] ; \mathbb{R}), \quad b \equiv 0$ are the skalar kernel functions and condition $\left(\mathbf{H}_{\alpha}\right)$ holds. If $\Psi \in \mathcal{P U C}\left([0, T] ; L^{2}(\Omega ; D(A))\right)$, then the following formula holds

$$
W^{\Psi}(t)=c A \int_{0}^{t} T(t-\tau) \times\left[\int_{0}^{\tau}(\dot{a}+c k+\dot{a} \times k)(\tau-\sigma) W^{\Psi}(\sigma) \mathrm{d} \sigma+c \int_{0}^{\tau} \Psi(s) \mathrm{d} W(s)\right] \mathrm{d} \tau+\int_{0}^{t} \Psi(\tau) \mathrm{d} W(\tau),
$$

where $t \in[0, T], \quad c=a(0) \neq 0$ is a constant and

like in Equation (1).

$$
\int_{0}^{t} \Psi(\tau) \mathrm{d} W(\tau)=\sum_{i=1}^{\infty} \int_{0}^{t} \Psi(\tau) \mathrm{d} W(\tau)
$$

Proof Since formula (9) holds for any bounded operator, then it holds for the Yosida approximation $A_{n}$ of operator $A$ as well, that is

$$
W_{n}^{\Psi}(t)=\int_{0}^{t}(a+a \times k)(t-\tau) A_{n} W_{n}^{\Psi}(\tau) \mathrm{d} \tau+\sum_{i=1}^{\infty} \int_{0}^{t} \Psi_{i}(\tau) \mathrm{d} W_{i}(\tau),
$$


where

$$
W_{n}^{\Psi}(t):=\sum_{i=1}^{\infty} \int_{0}^{t} R_{n}(t-\tau) \Psi_{i}(\tau) \mathrm{d} W_{i}(\tau)
$$

Denoting

$$
Z_{n}(t):=\int_{0}^{t}\left(a+a^{*} k\right)(t-\tau) W_{n}^{\Psi}(\tau) \mathrm{d} \tau, t \in[0, T]
$$

and using the Leibniz rule twice we obtain

$$
Z_{n^{\prime}}(t)=\int_{0}^{t}(\dot{a}+a(0) k+\dot{a} \times k)(t-\tau) W_{n}^{\Psi}(\tau) \mathrm{d} \tau+a(0) W_{n}^{\Psi}(t) .
$$

From (23) and (24), we can write

$$
W_{n}^{\Psi}(t)=A_{n} Z_{n}(t)+\int_{0}^{t} \Psi(\tau) \mathrm{d} W(\tau), t \in[0, T] .
$$

If $a(0) \neq 0$, then from (25) we have

$$
W_{n}^{\Psi}(t)=\frac{1}{a(0)}\left[Z_{n^{\prime}}(t)-\int_{0}^{t}(\dot{a}+a(0) k+\dot{a} \times k)(t-\tau) W_{n}^{\Psi}(\tau) \mathrm{d} \tau\right],
$$

and next

$$
Z_{n^{\prime}}(t)=a(0) A_{n} Z_{n}(t)+\int_{0}^{t}(\dot{a}+a(0) k+\dot{a} \times k)(t-\tau) W_{n}^{\Psi}(\tau) \mathrm{d} \tau+a(0) \int_{0}^{t} \Psi(\tau) \mathrm{d} W(\tau), t \in[0, T] .
$$

For simplicity, we introduce the following term

$$
\tilde{W}_{n}^{\Psi}(t):=\int_{0}^{t}(\dot{a}+a(0) k+\dot{a} \times k)(t-\tau) W_{n}^{\Psi}(\tau) \mathrm{d} \tau, t \in[0, T] .
$$

Then

$$
Z_{n^{\prime}}(t)=c A_{n} Z_{n}(t)+\left[\tilde{W}_{n}^{\Psi}(t)+c \int_{0}^{t} \Psi(\tau) \mathrm{d} W(\tau)\right] \text {, gdzie } c=a(0) .
$$

Since $Z_{n}(0)=0$, we can write

$$
Z_{n}(t)=\int_{0}^{t} \mathrm{e}^{c(t-\tau) A_{n}}\left[\tilde{W}_{n}^{\Psi}(\tau)+c \int_{0}^{\tau} \Psi(s) \mathrm{d} W(s)\right] \mathrm{d} \tau, t \in[0, T] .
$$

From (23) and (24) we obtain

$$
W_{n}^{\Psi}(t)=A J_{n} Z_{n}(t)+\int_{0}^{t} \Psi(\tau) \mathrm{d} W(\tau), t \in[0, T],
$$

where $J_{n}:=n \mathcal{R}(n, A)$.

Then

$$
W_{n}^{\Psi}(t)=A J_{n} \int_{0}^{t} \mathrm{e}^{c(t-\tau) A_{n}}\left[\tilde{W}_{n}^{\Psi}(\tau)+c \int_{0}^{\tau} \Psi(s) \mathrm{d} W(s)\right] \mathrm{d} \tau+\int_{0}^{t} \Psi(\tau) \mathrm{d} W(\tau), t \in[0, T] .
$$

Basing on Theorem 1, properties of Yosida approximation $A_{n}$ of the operator $A$, and dominated convergence theorem, we have

$$
\begin{aligned}
& \lim _{n \rightarrow \infty} J_{n} x=x, \text { for any } x \in H, \\
& \lim _{n \rightarrow \infty} A_{n} x=A x, \text { for any } x \in D(A),
\end{aligned}
$$




$$
\lim _{n \rightarrow \infty} \mathrm{e}^{t A_{n}} X=T(y) x, \text { for any } x \in H,
$$

and

$$
\lim _{n \rightarrow \infty} \sup _{t \in[0, T]} \mathbb{E}\left|W_{n}^{\Psi}(t)-W^{\Psi}(t)\right|_{H}^{2}=0 .
$$

Since the operator $A$ is closed, we can conclude that

$$
\int_{0}^{t} T(t-\tau)\left[\tilde{W}_{n}^{\Psi}(\tau)+c \int_{0}^{\tau} \Psi(s) \mathrm{d} W(s)\right] \mathrm{d} \tau \in D(A) .
$$

Hence, passing to the limit with $n \rightarrow \infty$, we obtain

$$
W^{\Psi}(t)=c A \int_{0}^{t} T(t-\tau)\left[\tilde{W}^{\Psi}(\tau)+c \int_{0}^{\tau} \Psi(s) \mathrm{d} W(s)\right] \mathrm{d} \tau+\int_{0}^{t} \Psi(\tau) \mathrm{d} W(\tau), t \in[0, T]
$$

where

$$
\tilde{W}^{\Psi}(\tau)=\int_{0}^{\tau}(\dot{a}+c k+\dot{a} \times k)(\tau-\sigma) W^{\Psi}(\sigma) \mathrm{d} \sigma, \tau \in[0, T] .
$$

Lemma 2 Let the assumptions of Theorem 8 hold and $a(0)=1$. Then for $t \in[0, T]$,

$$
W^{\Psi}(t)=A Y(t)+\int_{0}^{t} \Psi(\tau) \mathrm{d} W(\tau)
$$

where

$$
Y(t):=\int_{0}^{t} T(t-\tau)\left[\tilde{W}^{\Psi}(\tau)+\int_{0}^{\tau} \Psi(s) \mathrm{d} W(s)\right] \mathrm{d} \tau
$$

and

$$
\tilde{W}^{\Psi}(\tau)=\int_{0}^{\tau}(\dot{a}+k+\dot{a} \times k)(\tau-\sigma) W^{\Psi}(\sigma) \mathrm{d} \sigma, \tau \in[0, T] .
$$

Moreover, $Y \in C^{1}([0, T] ; D(A))$, $P-$ a.s. and

$$
\frac{\mathrm{d} Y(t)}{\mathrm{d} t}=A Y(t)+\left[\tilde{W}^{\Psi}(t)+\int_{0}^{t} \Psi(s) \mathrm{d} W(s)\right], t \in[0, T] .
$$

Proof The formula (26) results from (22) and the definition (27) of the process $Y$. Moreover, from properties of convolution $Y \in C^{1}([0, T] ; D(A))$, $P$ - a.s.

Using the Leibniz rule and property of semigroup we obtain

$$
\begin{aligned}
\frac{\mathrm{d} Y(t)}{\mathrm{d} t} & =\int_{0}^{t} \frac{\mathrm{d} T(t-\tau)}{\mathrm{d} t}\left[\tilde{W}^{\Psi}(\tau)+\int_{0}^{\tau} \Psi(s) \mathrm{d} W(s)\right] \mathrm{d} \tau+T(0)\left[\tilde{W}^{\Psi}(t)+\int_{0}^{t} \Psi(s) \mathrm{d} W(s)\right] \\
& =A \int_{0}^{t} T(t-\tau)\left[\tilde{W}^{\Psi}(\tau)+\int_{0}^{\tau} \Psi(s) \mathrm{d} W(s)\right] \mathrm{d} \tau+\left[\tilde{W}^{\Psi}(t)+\int_{0}^{t} \Psi(s) \mathrm{d} W(s)\right] \\
& =A Y(t)+\left[\tilde{W}^{\Psi}(t)+\int_{0}^{t} \Psi(s) \mathrm{d} W(s)\right],
\end{aligned}
$$

where $t \in[0, T]$.

To conclude continuity of trajectories of stochastic convolution, we use regularity of solutions for the nonhomogeneous Cauchy problem [7] to the formula (26).

Theorem 9 Suppose that the assumptions of Theorem 8 hold. If both processes $Y$ and $\int_{0}^{t} \Psi(\tau) \mathrm{d} W(\tau)$, 
$t \in[0, T]$, have continuous trajectories in the space $D_{A}(\alpha, \infty)$, then the stochastic convolution $W^{\Psi}(t)$, $t \in[0, T]$, has continuous trajectories in $D_{A}(\alpha, \infty)$.

In the previous theorem, the space $D_{A}(\alpha, \infty), \alpha \in(0,1)$, is defined as follows. For any $\alpha \in(0,1)$, we set

$$
|x|_{\alpha, \infty}:=\sup _{t>0} \frac{|T(t) x-x|}{t^{\alpha}}, \quad x \in H .
$$

We denote by $D_{A}(\alpha, \infty)$ the Banach space of all $x \in H$ such that $|x|_{\alpha, \infty}<\infty$, endowed with the norm $\|_{H}+||_{\alpha, \infty}$. By the interpolation theory, $D_{A}(\alpha, \infty)$ is an invariant space of $T(t), t>0$, and the restriction of $T(t)$ to $D_{A}(\alpha, \infty)$ generates a $\mathcal{C}_{0}$-semigroup in $D_{A}(\alpha, \infty)$.

\section{Analogue of the Itô Formula}

In this section, we derive the analogue of the Itô formula to the perturbed Volterra Equation (1).

Proposition 10 Let the process $X$ be a strong solution to the Equation (1) and $a, \dot{a}, k, b, \dot{b} \in L_{l o c}^{1}([0, T] ; \mathbb{R})$. Suppose that the function $F:[0, T] \times H \rightarrow \mathbb{R}$ and its partial derivatives $F_{t}, F_{x}, F_{x x}$ are uniformly continuous on $[0, T] \times H$. Then, for any $t \in[0, T]$,

$$
\begin{aligned}
& F(t, X(t))-F(0, X(0)) \\
& =\int_{0}^{t} F_{t}(\tau, X(\tau)) \mathrm{d} \tau+\int_{0}^{t}\left\langle F_{x}(\tau, X(\tau)),(a+a \times k)(0) A X(\tau)\right\rangle_{H} \mathrm{~d} \tau \\
& +\int_{0}^{t}\left\langle F_{x}(\tau, X(\tau)),((\dot{a}+a(0) k+\dot{a} \times k) \times A X)(\tau)\right\rangle_{H} \mathrm{~d} \tau+\int_{0}^{t}\left\langle F_{X}(\tau, X(\tau)), b(0) X(\tau)+(\dot{b} \times X)(\tau)\right\rangle_{H} \mathrm{~d} \tau \\
& +\sum_{i=1}^{\infty} \int_{0}^{t}\left\langle F_{X}(\tau, X(\tau)), \Psi_{i}(\tau) \mathrm{d} W_{i}(\tau)\right\rangle_{H}+\frac{1}{2} \int_{0}^{t} \sum_{i=1}^{\infty}\left\langle F_{x x}(\tau, X(\tau)) \Psi_{i}(\tau), \Psi_{i}(\tau)\right\rangle_{H} \mathrm{~d} \tau, P-\text { a.s. }
\end{aligned}
$$

The following proposition is an example of application of the above analogue of the It formula.

Proposition 11 Let the operator A be the generator of bounded analytic semigroup in H. Suppose that $a, \dot{a}, k, b, \dot{b} \in L_{\text {loc }}^{1}([0, T] ; \mathbb{R})$, the conditions $\left(\mathbf{H}_{\alpha}\right)$ and $\left(\mathbf{H}_{\beta}\right)$ hold and $\Psi \in \mathcal{P U C}\left([0, T] ; L^{2}(\Omega ; D(A))\right)$.

Assume that the function $v: H \rightarrow \mathbb{R}$ satisfies the following conditions:

1) function $v$ and its partial derivatives $v_{x}, v_{x x}$ are uniformly continuous on bounded subsets of $H$,

2) for any $X \in D(A)$ and the constant $c>0$

$$
|v(X)|+|X|_{H}\left|v_{x}(X)\right|_{H}+|X|_{H}^{2}\left|v_{x x}(X)\right|_{H} \leq c|X|_{H}^{2},
$$

3) for any $X \in D(A)$ and the constant $\alpha \in \mathbb{R}$

$$
\begin{aligned}
\alpha v(X) \leq & -\left\langle v_{x}(X),(a+a \times k)(0) A X(t)+((\dot{a}+a(0) k+\dot{a} \times k) \times A X)(t)+b(0) X(t)+(\dot{b} \times X)(t)\right\rangle_{H} \\
& -\frac{1}{2} \sum_{i=1}^{\infty}\left\langle v_{x x}(X) \Psi_{i}(t), \Psi_{i}(t)\right\rangle_{H} .
\end{aligned}
$$

Then the stochastic convolution $W^{\Psi}$ satisfies the following inequality

$$
\mathbb{E} v\left(W^{\Psi}(t)\right) \leq \mathrm{e}^{-\alpha t} v(0) .
$$

The idea of the proof bases on Ichikawa's scheme, see ([8], Theorem 3.1), and on Theorem 1 and Proposition 10. It seems to be a good starting point in the study of stability of mild solution to perturbed Volterra Equation (1).

\section{References}

[1] Da Prato, G. and Zabczyk, J. (1992) Stochastic Equations in Infinite Dimensions. Cambridge University Press, Cambridge. http://dx.doi.org/10.1017/CBO9780511666223

[2] Karczewska, A. (2007) Properties of Convolutions Arising in Stochastic Volterra Equations. International Journal of Contemporary Mathematical Sciences, 2, 1037-1051. 
[3] Karczewska, A. (2007) Convolution Type Stochastic Volterra Equations. Lecture Notes in Nonlinear Analysis. Juliusz Schauder Center for Nonlinear Studies. 10. Toruń.

[4] Karczewska, A. and Lizama, C. (2009) Strong Solutions to Stochastic Volterra Equations. Journal of Mathematical Analysis and Applications, 349, 301-310. http://dx.doi.org/10.1016/j.jmaa.2008.09.005

[5] van Gaans, O. (2005) A Series Approach to Stochastic Differential Equations with Infinite Dimensional Noise. Integral Equations and Operator Theory, 51, 435-458. http://dx.doi.org/10.1007/s00020-003-1258-4

[6] Karczewska, A. and Lizama, C. (2014) Stochastic Volterra Equations under Perturbations. Electronic Communications in Probability, 19, 1-14. http://dx.doi.org/10.1214/ECP.v19-3365

[7] Da Prato, G. and Grisvard, P. (1979) Equations d'volution abstraites non linaires de type parabolique. Annali di Matematica Pura ed Applicata, 120, 329-396. http://dx.doi.org/10.1007/BF02411952

[8] Ichikawa, A. (1982) Stability of Semilinear Stochastic Evolution Equations. Journal of Mathematical Analysis and Applications, 90, 12-44. http://dx.doi.org/10.1016/0022-247X(82)90041-5 